Check for updates

Cite this: RSC Adv., 2017, 7, 34746

Received 10th May 2017

Accepted 4th July 2017

DOI: 10.1039/c7ra05291j

rsc.li/rsc-advances

\title{
A simple, fast, and sensitive colorimetric assay for visual detection of berberine in human plasma by $\mathrm{NaHSO}_{4}$-optimized gold nanoparticles $\uparrow$
}

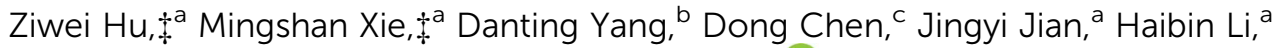 \\ Kaisong Yuan, ${ }^{a}$ Zhengjin Jiang ${ }^{* a}$ and Haibo Zhou (D) *a
}

\begin{abstract}
Berberine is an important anti-inflammatory drug widely used for the treatment of heart and intestinal disorders. The quantification of berberine is usually required in clinic medical assays. Herein, we report simple, fast and sensitive colorimetric visualization of berberine in human plasma using citrate-stabilized

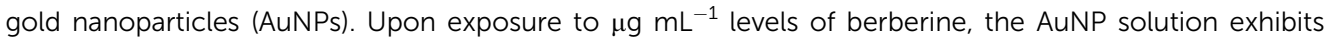
a highly sensitive color change from red to blue and rapid aggregation kinetics within the initial $10 \mathrm{~min}$, which can be directly seen with the naked eye and monitored by UV-vis absorbance spectroscopy. As confirmed by comparison with potential coexisting foreign substances, the presence of positively charged berberine induces the aggregation of the AuNPs owing to the electrostatic attraction and coordination interactions between the ring nitrogen atoms of berberine and the AuNPs, causing a color change of the AuNP suspension. Further evidence is that the sensitivity is significantly improved when $\mathrm{NaHSO}_{4}$ is added to promote the ligand exchange between citrate and berberine at the surface of the AuNPs. The $\mathrm{NaHSO}_{4}$-optimized AuNP system provides a simple colorimetric assay for the rapid detection of berberine in human plasma. The limit of detection (LOD) is $0.24 \mu \mathrm{g} \mathrm{mL}^{-1}$ by the naked eye and $0.06 \mu \mathrm{gL}^{-1}$ by UV-vis spectroscopy.
\end{abstract}

\section{Introduction}

Berberine is an isoquinoline alkaloid isolated from many medicinal herbs, such as Rhizoma coptidis, Berberis aquifolium, and Berberis vulgaris. ${ }^{1}$ It has been extensively used as an important anti-inflammatory drug for heart and intestinal disorders for many years, owing to its antitumor promotion activity and anti-lipase effect. ${ }^{2}$ During the last few decades, many studies have shown that berberine can be used as a novel cholesterol-lowering drug, and as an anti-diabetes agent. ${ }^{3}$ It also has various beneficial effects on the cardiovascular system, ${ }^{4}$ and significant antioxidant activities. ${ }^{5,6}$ In addition, the potential application of berberine as a complementary therapeutic agent for HIV infection has also been reported. ${ }^{7}$ Thus, it is important to study the pharmacokinetics of berberine for its potential

anstitute of Pharmaceutical Analysis, College of Pharmacy, Jinan University, Guangzhou, Guangdong 510632, China. E-mail: haibo.zhou@jnu.edu.cn; jzjjackson@hotmail.com

${ }^{b}$ Department of Preventative Medicine, Zhejiang Provincial Key Laboratory of Pathological and Physiological Technology, Medical School of Ningbo University, Ningbo, Zhejiang 315211, China

'Department of Urology, Sun Yat-sen University Cancer Center, Guangzhou, Guangdong 510060, China

$\dagger$ Electronic supplementary information (ESI) available. See DOI: $10.1039 / \mathrm{c} 7 \mathrm{ra0} 2291 \mathrm{j}$

$\ddagger$ These authors equally contributed to this work. systemic therapeutic effects. In order to achieve this goal, it is necessary to establish a rapid, simple, and sensitive method for the detection of berberine in human plasma. Until now, several methods have been developed for the detection of berberine, such as HPLC, ${ }^{8}$ ultra-performance liquid chromatographyelectrospray ionization-mass spectrometry (UPLC-ESI-MS/ MS), ${ }^{9,10}$ spectrofluorometric methods ${ }^{11}$ fluorescent aptasensors, ${ }^{12}$ and optical fiber sensing with integrating sphere reflection. ${ }^{13}$ These methods all have the advantages of high sensitivity and selectivity, but require expensive instruments or complicated procedures, making on-site and fast visual berberine sensing difficult.

Compared with the above methods for berberine detection, colorimetric sensors based on noble metal nanoparticles have attracted more and more attention due to their distinct variation in color associated with morphology changes. Colorimetric visualization assays can achieve the rapid, reliable and sensitive detection of many analytes, such as melamine, ${ }^{14,15}$ small molecules $^{16}$ and heavy metal ions, ${ }^{17}$ without the use of costly instrumentation or the need for tedious training. Recently, Jian Ling et al. ${ }^{18}$ reported a colorimetric method for the detection of berberine hydrochloride based on the color change of aggregated silver nanoparticles (AgNPs). But the results they obtained were not satisfactory, as the color change of the AgNPs in response to different concentrations of berberine was not obvious enough to be recognized by the naked eye due to the 
intrinsic optical properties of the system. In addition, they didn't use the developed method for real sample detection. As a result of surface plasma resonance (SPR), AuNPs exhibit sizetunable optical properties. ${ }^{19}$ AuNPs have bright colors in the visible spectral range concerning their SPR absorption and light scattering. Colloidal AuNPs are wine-red, whereas their aggregates appear purple or blue in color. The controlled color change induced by aggregation can form the basis of a colorimetric assay. Taking this into account, we used unmodified gold nanoparticles (AuNPs) as a probe for the detection of berberine in human plasma. Citrate-capped AuNPs have an electronegatively charged surface and can be dispersed from each other in water symmetrically by electrostatic repulsion, exhibiting red color for the plasmon resonance absorption. ${ }^{20}$ However, the presence of positively charged berberine would induce the aggregation of the AuNPs owing to electrostatic attraction, causing a color change of the AuNP suspension. In addition, as an alkaloid, the berberine molecule contains ring nitrogen atoms with a strong binding ability to the surface of AuNPs. The AuNPs can thus be crosslinked directly in the presence of certain amounts of berberine without any extra aid.

Based on the above considerations, we demonstrate herein that the color change induced by the electrostatic and coordination interactions between berberine and the surface of AuNPs can be used for the rapid detection of berberine. Since such a color change can be readily seen by the naked eye, the method demonstrated here enables on-site and visual detection of berberine in human plasma without the aid of any advanced instruments. With this colorimetric sensor, we could identify trace levels of berberine in human plasma within $10 \mathrm{~min}$, as measured with the naked eye or UV-vis spectroscopy.

\section{Experimental}

\section{Materials}

Berberine (purity > 97\%), chloroauric acid tetrahydrate $\left(\mathrm{HAuCl}_{4} \cdot 4 \mathrm{H}_{2} \mathrm{O}\right)$ and trisodium citrate were purchased from Macklin. Tetrahydropapaverine hydrochloride, tetrahydropalmatine and isoquinoline were supplied by Aladdin. These materials were untreated and directly used. Human plasma was provided by Guangdong Provincial Hospital of Traditional Chinese Medicine. Ultra-pure water was supplied by a Millipore water purification system. All glass instruments were washed with concentrated hydrochloric acid and concentrated nitric acid in a volume ratio of $3: 1$ before use, and then washed with ultra-pure water and dried.

\section{Synthesis of AuNPs}

AuNPs were prepared by the reduction of $\mathrm{HAuCl}_{4}$ with trisodium citrate. Typically, $0.25 \mathrm{~mL}$ of $\mathrm{HAuCl}_{4} \cdot 4 \mathrm{H}_{2} \mathrm{O}(0.1 \mathrm{M})$ was added to $100 \mathrm{~mL}$ of ultra-pure water and heated to boiling under reflux with magnetic stirring. $1.5 \mathrm{~mL}$ of trisodium citrate (1\%) was rapidly added into the boiling solution of $\mathrm{HAuCl}_{4}$, and the mixed solution was further refluxed for another $30 \mathrm{~min}$ to form a wine-red suspension. The suspension was gradually cooled to room temperature under stirring, and then filtered through a $0.22 \mu \mathrm{m}$ Millipore membrane. The filtrate was stored in a refrigerator at $4{ }^{\circ} \mathrm{C}$ for further use. The average size of the AuNPs is $18 \mathrm{~nm}$ as examined by transmission electron microscopy. The concentration of the AuNPs as determined by UV-vis spectrometry was $0.30 \mathrm{nM}$. The AuNPs were directly used for all further studies at the concentration of $0.30 \mathrm{nM}$ without further concentration or dilution.

\section{Detection of berberine}

Typically, $10 \mathrm{~mL}$ of AuNP suspension was used as a stock liquid for the detection of berberine. Different amounts of analyte were added into $2 \mathrm{~mL}$ of the above AuNP suspension. The color change and absorbance spectra were observed with the naked eye and recorded with a UV-vis spectrometer, respectively. The aggregation kinetics of the AuNPs at different concentrations of the berberine analyte were obtained by measurement of the absorbance spectra at intervals of $2.5 \mathrm{~min}$. On the other hand, the sensitivity of the AuNP suspension to berberine was further optimized with $\mathrm{NaHSO}_{4}$. Typically, $9.5 \mathrm{~mL}$ of the original AuNP suspension was diluted with $10 \mathrm{~mL}$ of deionized water and 0.5 $\mathrm{mL}$ of $4.0 \mathrm{mM} \mathrm{NaHSO}_{4}$ to give a mixture with a total volume of $20 \mathrm{~mL}$. The AuNP suspension containing $0.1 \mathrm{mM} \mathrm{NaHSO}_{4}$ was used as a stock liquid for the detection of berberine in real human plasma samples.

\section{Extracts of real samples}

$100 \mu \mathrm{L}$ of berberine $(1 \mathrm{mM})$ was doped into $500 \mu \mathrm{L}$ of human plasma to validate the effectiveness of the colorimetric detection of berberine in real samples. The extraction of human plasma for the determination of berberine was carried out according to the following method. Typically, $100 \mu \mathrm{L}$ of berberine solution $(1 \mathrm{mM})$ was added into $500 \mu \mathrm{L}$ of plasma, and the mixture was vortexed for 30 seconds. Then, $3 \mathrm{~mL}$ of chloroform was added. The mixture was vortexed for 2 minutes and centrifuged at $4000 \mathrm{rpm}$ for 10 minutes. The organic phase was transferred to a centrifuge tube and evaporated to dryness at $40{ }^{\circ} \mathrm{C}$ under a gentle stream of nitrogen. The residue was redissolved with $100 \mu \mathrm{L}$ of mobile phase consisting of acetonitrile and water $(33: 67, \mathrm{v} / \mathrm{v})$, then vortexed for 30 seconds, sonicated for 10 minutes, and centrifuged at $4000 \mathrm{rpm}$ for 5 minutes. The supernatant was used as the final sample solution. The extract contained $169.77 \mu \mathrm{g} \mathrm{mL} \mathrm{m}^{-1}$ berberine according to the measurement by high-performance liquid chromatography, and was diluted five times for the colorimetric assay.

\section{Characterization}

UV-vis spectra were recorded with a Varian Carry-5000 UV-VisNIR spectrophotometer using $1 \mathrm{~cm}$ path length quartz cuvettes. The zeta potential of the AuNPs was recorded with a Malvern Zetasizer Nano-ZS. The AuNPs were observed using a JEM-2100F transmission electron microscope. Optical photographs were taken with a Canon camera. The concentration of berberine in the sample solution was measured using an Agilent 1100 high-performance liquid chromatography system with a UV detector. 


\section{Results and discussion}

Electrostatic attraction and coordination interaction-based aggregation mechanism for the sensing system

Typically, colloidal solutions of AuNPs are wine-red. Once aggregation of the AuNPs occurs, the UV-vis absorbance of the AuNPs changes obviously due to the decrease in the interparticle distance, causing a color change of the AuNP solution. The citrate-capped AuNPs prepared in this contribution are electronegatively charged (Fig. $\mathrm{S} 1 \mathrm{~A} \dagger$ ), and dispersed from each other symmetrically by the electrostatic repulsion of each particle. The aggregation of the AuNPs occurred when electropositively charged berberine was mixed with the colloidal solution of AuNPs, which is due to the neutralization of the electrostatic repulsion on each gold nanoparticle (Fig. S1B $\dagger$ ). The surface charge of the AuNPs changes from $-47.2 \mathrm{mV}$ to $-26.5 \mathrm{mV}$. Furthermore, berberine with a nitrogen hybrid ring as a binding site may strongly coordinate to the AuNPs by ligand exchange with weakly surface-bound citrate ions, and finally crosslink the AuNPs. Thus, the colloidal stability was drastically reduced, resulting in the prompt occurrence of particle aggregation, as revealed in Fig. 1. The electrostatic attraction and coordination interaction-based double aggregation mechanism offers a reliable approach to a simple and rapid colorimetric assay for the detection of berberine in human blood, which does not require any extra aid such as specific acceptors.

\section{Colorimetric sensitivity of the AuNP suspension to trace berberine}

In this study, $18 \mathrm{~nm}$ AuNPs were first synthesized by the reduction of $\mathrm{HAuCl}_{4}$ with sodium citrate in aqueous solution based on the reported literature. ${ }^{21}$ The resulting citratestabilized AuNPs were wine-red, because of their strong SPR at $520 \mathrm{~nm}$. Upon direct exposure of the citrate-stabilized AuNPs to berberine, the color of the AuNPs changed from wine-red, to purple to blue progressively as the berberine concentration

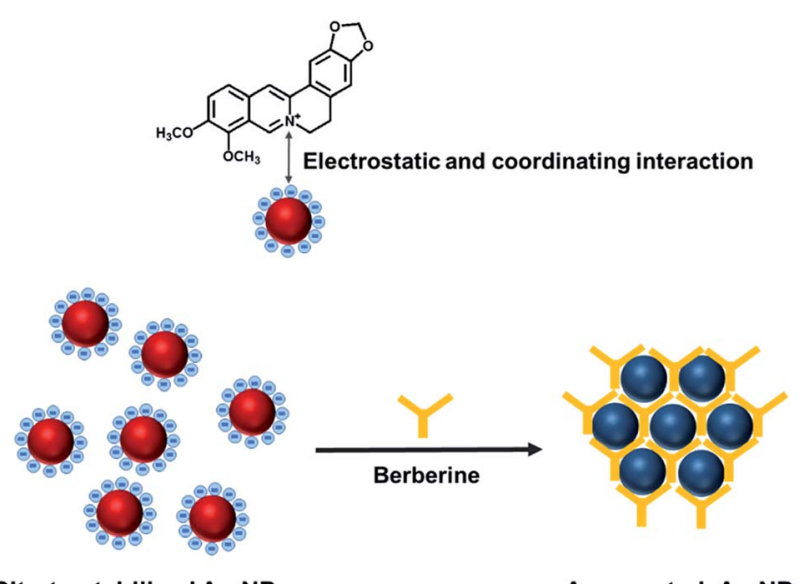

Citrate-stabilized Au NPs

Aggregated Au NPs

Fig. 1 Electrostatic attraction and coordination interaction between berberine and AuNPs, which causes the aggregation of the citratestabilized AuNPs. increased from 0 to $4.5 \mu \mathrm{M}$ (Fig. 2A). Clear color changes were observed from wine-red to deep red at a berberine concentration as low as $2.5 \mu \mathrm{M}$. Meanwhile, the berberine-induced aggregation of the AuNPs was also monitored by UV-vis spectroscopy (Fig. 2B). With the addition of berberine from 0 to 4.5 $\mu \mathrm{M}$, the original absorbance of the AuNPs at $520 \mathrm{~nm}$ decreased gradually, while a new absorbance (centered at $670 \mathrm{~nm}$ ) from the resulting aggregates increased obviously. This indicates that more and more monodisperse AuNPs were consumed to form more and more aggregates, which was further confirmed by the TEM observations, which showed monodisperse nanoparticles in the absence of berberine (Fig. 2C) and significant aggregation of the nanoparticles in the presence of 3 and $5 \mu \mathrm{M}$ berberine (Fig. 2D and E, respectively). These above observations clearly indicate that trace levels of berberine can directly induce the aggregation of unmodified AuNPs. Furthermore, the corresponding colorimetric effect was evaluated using the $A_{670} / A_{520}$ ratio in the presence of different concentrations of berberine (Fig. 3). The very small ratios reveal a slight visible color change from wine-red to deep red in the berberine concentration range of $0.5-2.5 \mu \mathrm{M}$, and the large ratios show color mutation from deep red to blue with a further increase in the concentration of berberine. The extinction ratios slightly increased from 0.10 to 0.23 in the berberine concentration range of $0-2.0 \mu \mathrm{M}$. The ratios of $A_{670} / A_{520}$ for 3.0, 3.5 and $4.0 \mu \mathrm{M}$ berberine are 1.09, 1.34 and 1.35 , respectively. As indicated by the arrow in Fig. 3 , the mutation point of the color is at $2.0 \mu \mathrm{M}$ berberine. The measurements of the extinction ratios further confirm that particle aggregation and a corresponding color change occur before the berberine concentration increases up to $2.5 \mu \mathrm{M}$.

\section{Aggregation kinetics of AuNPs with berberine}

We examined the aggregation kinetics of the unmodified AuNPs in the presence of trace levels of berberine by measuring the temporal evolution of $A_{670} / A_{520}$ at intervals of $2.5 \mathrm{~min}$. Because a high concentration of berberine $(>4.5 \mu \mathrm{M})$ leads to the rapid precipitation of the AuNPs during the mixing of berberine with the AuNPs, the aggregation kinetics cannot be accurately characterized by UV-vis spectroscopy. Therefore, we chose four typical concentrations of berberine $(1.0,2.0,2.5$ and $3.0 \mu \mathrm{M})$ for the evaluation of the aggregation kinetics. The time course of the aggregation of AuNPs and the plots of $A_{670} / A_{520}$ versus time at different berberine concentrations are shown in Fig. $\mathrm{S} 2 \dagger$ and 4, respectively. As shown in Fig. 4, a higher concentration of berberine caused a faster extinction ratio rise in the initial stage. At a concentration of $3.0 \mu \mathrm{M}$ berberine, the extinction ratio exhibits a rapid increase from the original value of 0.05 to 0.84 during the first $5 \mathrm{~min}$, followed by a slow increase to a maximum value of 1.38 after $40 \mathrm{~min}$, revealing that most of the free monodisperse AuNPs in the suspension are promptly consumed in the initial stage. The inset of Fig. 4 shows that the wine-red color of the AuNPs promptly changes into light purple after $5 \mathrm{~min}$, purple after $15 \mathrm{~min}$ and finally blue after $45 \mathrm{~min}$. The corresponding extinction ratios are about $0.84,1.17$ and 1.38 , respectively. In the case of $2.0 \mu \mathrm{M}$ berberine, however, the extinction ratio slightly increases from 0.05 to 0.11 in the first 


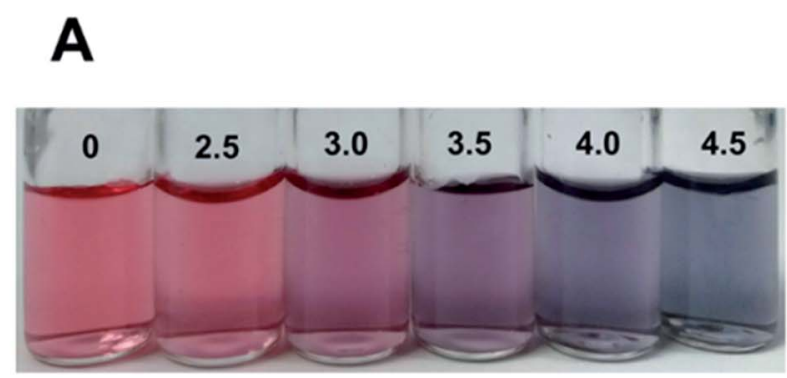

Concentration $(\mu \mathrm{M})$
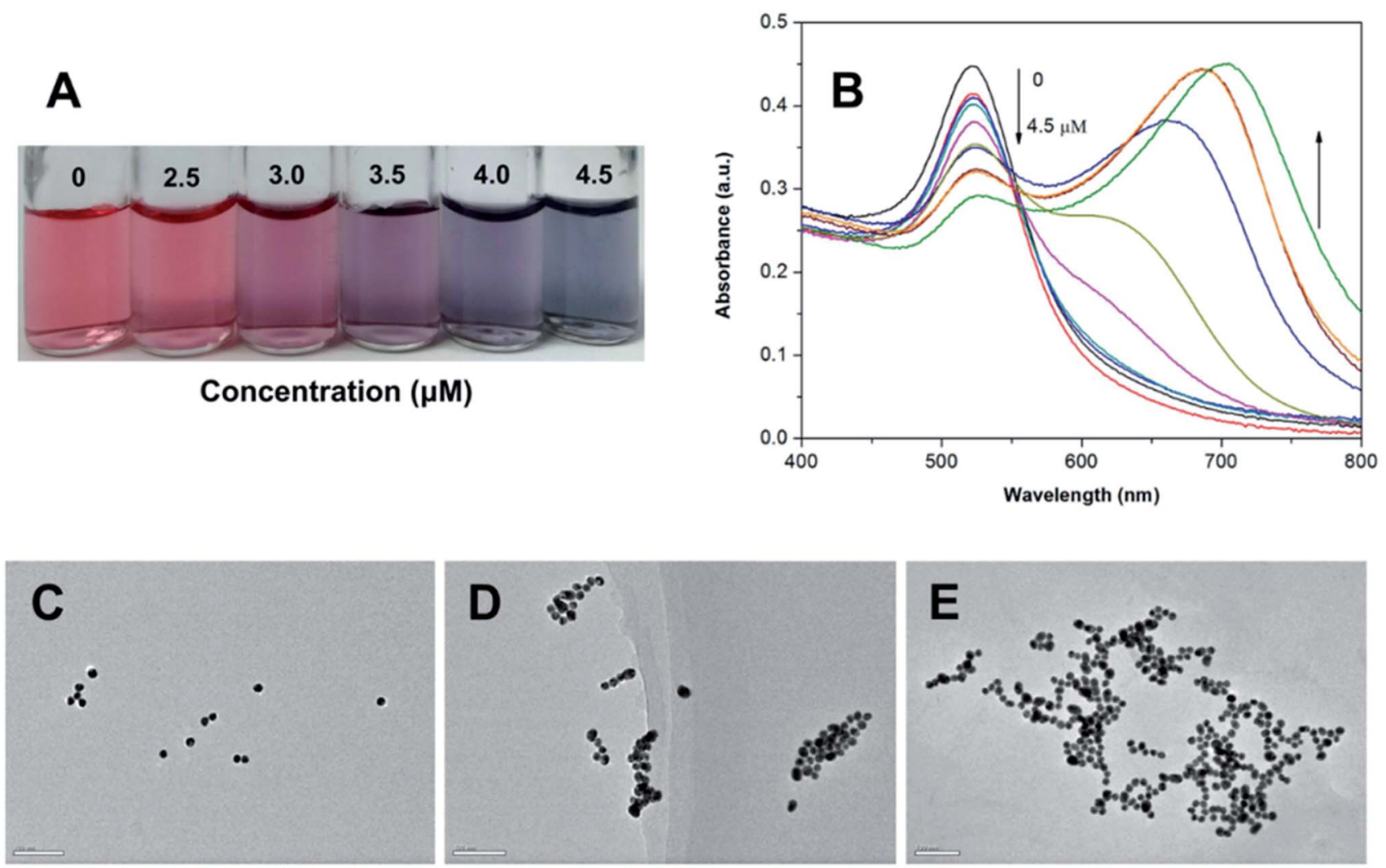

Fig. 2 (A) Visual color change of AuNPs with the indicated concentrations of berberine. (B) Evolution of the UV-vis absorbance spectra of the AuNP suspension with different concentrations of berberine: $0,0.5,1.0,1.5,2.0,2.5,3.0,3.5,4.0$, and $4.5 \mu M$. TEM images of AuNPs with the addition of (C) 0 , (D) 3.0, and (E) $5.0 \mu \mathrm{M}$ berberine, respectively. The scale bar is $100 \mathrm{~nm}$.

$2.5 \mathrm{~min}$, and then exhibits a linear enhancement to 0.47 in the next $55 \mathrm{~min}$, suggesting slow crosslinking aggregation of the AuNPs. When the concentration of berberine was further reduced to $1.0 \mu \mathrm{M}$, the extinction ratio very slightly increased in the first $2.5 \mathrm{~min}$ and then almost remained at a small constant

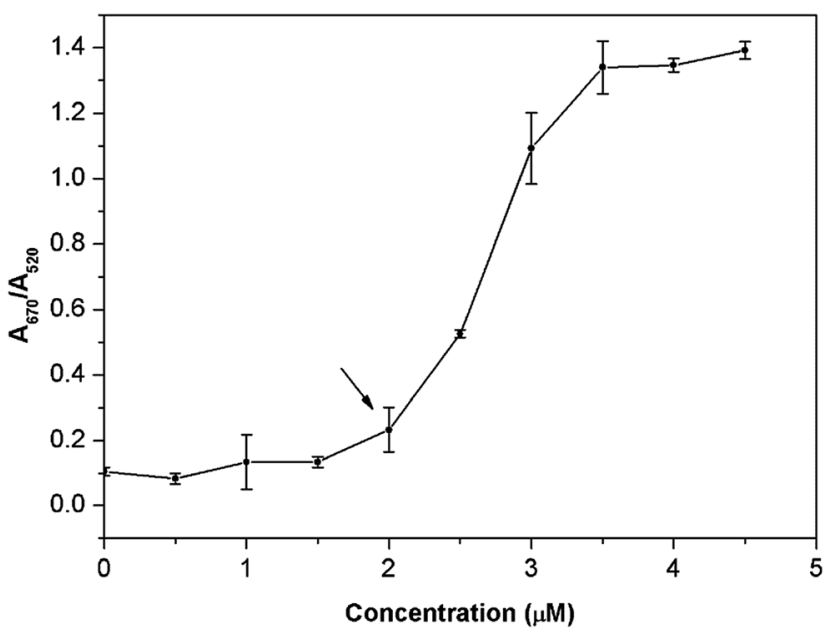

Fig. 3 Corresponding plot of $A_{670} / A_{520}$ versus berberine concentration at $0,0.5,1.0,1.5,2.0,2.5,3.0,3.5,4.0$, and $4.5 \mu \mathrm{M}$. All data were collected after $10 \mathrm{~min}$. The arrow indicates the mutation point of the color. value. This shows that the free berberine molecules were almost exhausted in a shorter time, but this was not enough to cause the significant aggregation of the AuNPs. In general, the aggregation of the AuNPs starts from the electrostatic

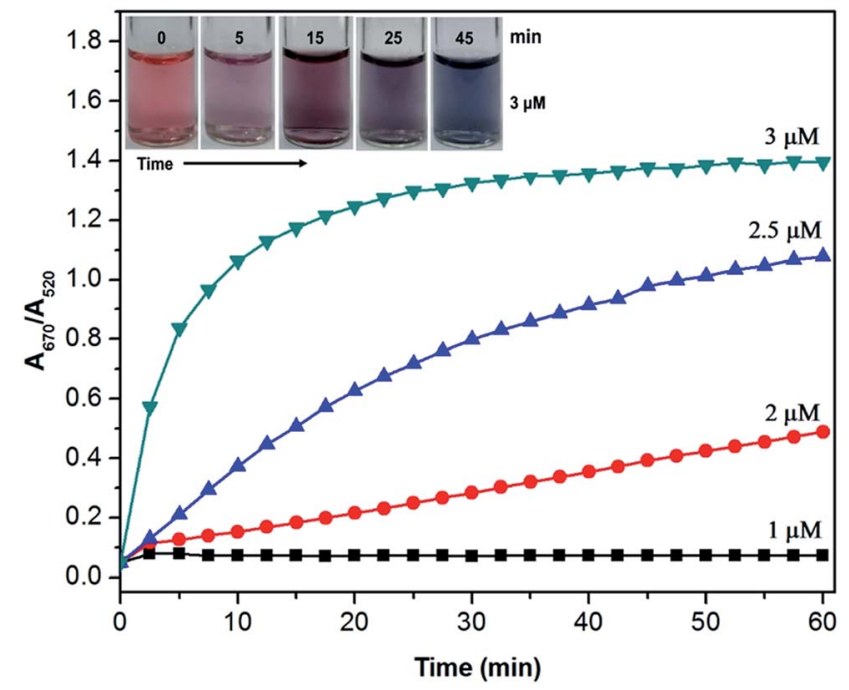

Fig. 4 Plots of $A_{670} / A_{520}$ versus time at different concentrations of berberine (the data were taken at intervals of $2.5 \mathrm{~min}$ ). The inset images show the color change of the AuNP suspension with time after the addition of $3.0 \mu \mathrm{M}$ berberine. 
interaction between berberine and the surface of the AuNPs, since the citrate-capped AuNPs prepared in this contribution are electronegatively charged, and dispersed from each other symmetrically by the electrostatic repulsion of each particle. The aggregation of the AuNPs occurred when electropositively charged berberine was mixed with the colloidal solution of AuNPs owing to the neutralization of the electrostatic repulsion from each gold nanoparticle. ${ }^{18}$ The free berberine molecules were promptly exhausted at concentrations lower than $2.0 \mu \mathrm{M}$ due to the strong adsorption at the surface of the AuNPs. In contrast, free AuNPs in the suspension were promptly consumed when the concentration of berberine was higher than $2.5 \mu \mathrm{M}$. These detailed observations further suggest that berberine has a strong electrostatic attraction ability to AuNPs without the aid of a salt to induce aggregation of the gold nanoparticles.

\section{Colorimetric selectivity}

To better understand the berberine-induced aggregation mechanism, we evaluated the colorimetric selectivity of the citrate-stabilized AuNPs using coexisting foreign substances, such as common metal ions, proteins, amino acids and structural analogues of berberine in a 96-well microplate (Fig. 5A). For three typical common compounds, no visible color change of the AuNPs was observed with the addition of $1 \mathrm{mM}$ starch (a), alanine (b) and glycine (c). Based on a multiple-interaction model accounting for specific chemical bonding between BSA and the AuNPs due to the presence of thiol, amine, and imidazole groups in BSA (i.e., cysteine, lysine, and histidine residues), ${ }^{22}$ addition of $5 \mu \mathrm{M}$ BSA (d) can result in a light purple color. Although also positively charged, common metal ions can only cause a color change of the AuNPs at a very high concentration. Purple color was only observed with the addition of 500 $\mu \mathrm{M} \mathrm{Ca}^{2+}$ (e) and $\mathrm{Mg}^{2+}$ (f). The results show that electrostatic interactions are not the only mechanism of AuNP aggregation, and the structure of berberine also plays an important role in the process of crosslinking of the AuNPs. Thus, we further investigated the effect of structural analogues of berberine on the aggregation of the AuNPs. The addition of $10 \mu \mathrm{M}$ tetrahydropapaverine (i) can result in a purple color. Furthermore, isoquinoline $(\mathrm{j})$ and tetrahydropalmatine $(\mathrm{k})$ at concentrations of $10 \mu \mathrm{M}$ can transform the wine-red AuNPs into blue and deep purple, respectively. These results indicated that the ring nitrogen atoms in these structural analogues of berberine exhibited strong coordination interaction with the AuNPs. It is worth noting that only $2.0 \mu \mathrm{M}$ of berberine (l) is sufficient to promptly transform the wine-red AuNPs into purple. Therefore, the sensitivity of the AuNPs to the above compounds is in the order of $\mathrm{l}>\mathrm{j} \approx \mathrm{d}>\mathrm{k}>\mathrm{i}>\mathrm{f}>\mathrm{h}>\mathrm{g} \approx \mathrm{a}, \mathrm{b}, \mathrm{c}$, and e. It is clear that the high sensitivity of the AuNPs to berberine is attributed here to effects of electrostatic interactions and coordination interactions to crosslink the AuNPs strongly via ring nitrogen atoms with the AuNPs, as illustrated in Fig. 1. The colorimetric selectivity was further evaluated by testing the UV-vis spectroscopic response of the AuNPs to the above compounds at $5 \mu \mathrm{M}$ in solution (Fig. 5B). The very small $A_{670} / A_{520}$ ratios for a, b, c, e
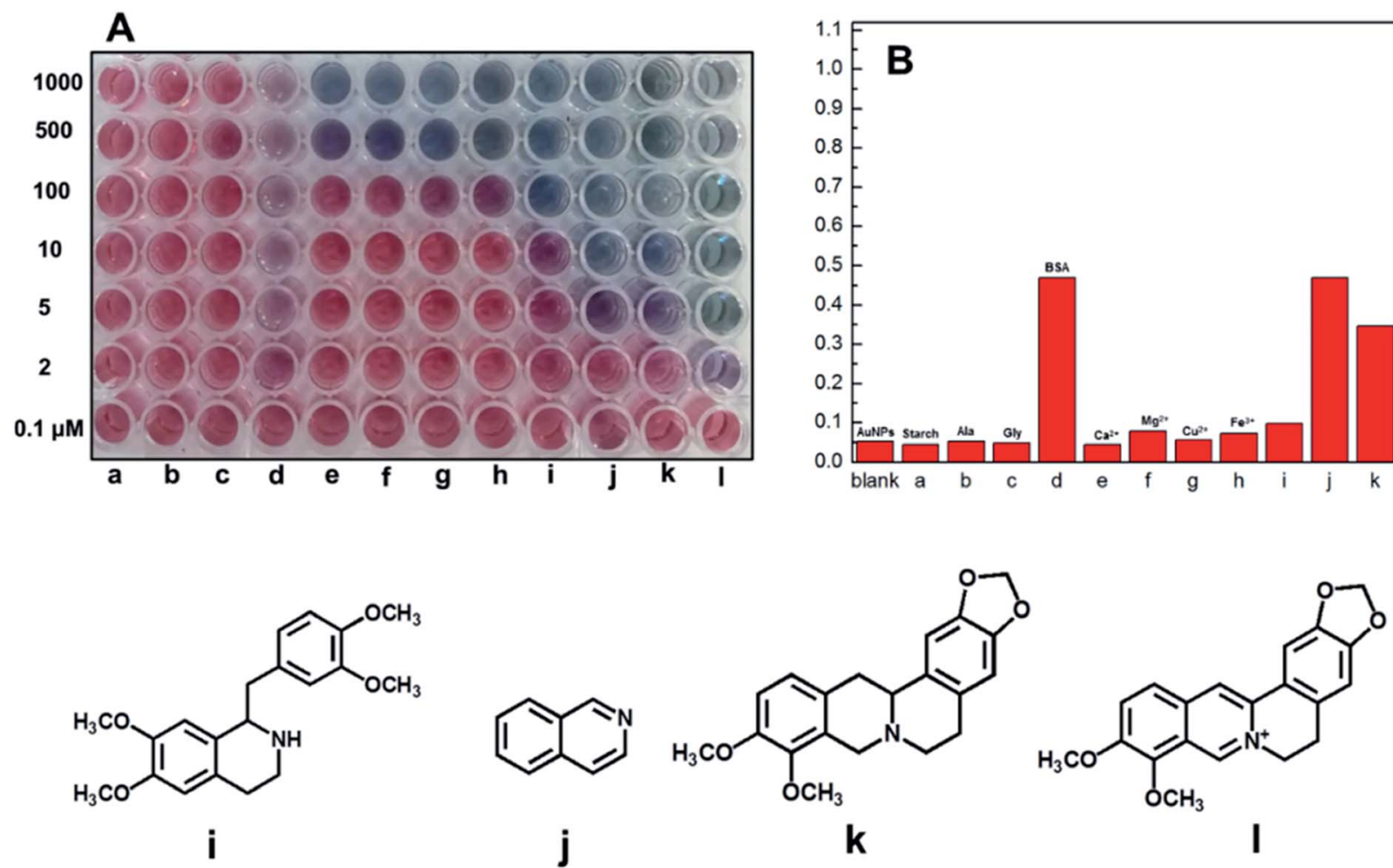

Fig. 5 (A) Colors of the unmodified AuNP suspension (in a 96-well microplate) after the addition of several compounds with different concentrations. (B) The corresponding $A_{670} / A_{520}$ ratios of the AuNP suspension with the addition of $5 \mu \mathrm{M}$ analytes. 
and $g$ are almost identical to that of blank AuNPs $(<0.05)$, suggesting no obvious aggregation of the AuNPs. Berberine exhibits the highest $A_{670} / A_{520}$ value (1.03), which is 2.94 fold more when compared with its most similar compound $\mathrm{k}(0.35)$. These quantitative measurements further show that both the positive charge and the ring nitrogen atoms in berberine or in its structural analogues $(\mathrm{i}, \mathrm{j}, \mathrm{k})$ induce the aggregation of the AuNPs cooperatively, as shown in Fig. 1.

\section{Optimized sensitivity with sodium bisulfate through surface ligand exchange}

To further confirm the aggregation mechanism and to improve the colorimetric sensitivity, we tested the effect of $\mathrm{NaHSO}_{4}$ as a promoter of ligand exchange between citrate and berberine at the surface of the AuNPs. Although the citrate ligands introduced in the synthesis can weakly bind to the surface of the AuNPs as a stabilizer, ${ }^{23}$ their relatively large molecular size may hinder the adsorption of berberine to the AuNPs and the further crosslinking of the AuNPs. It is well-known that $\mathrm{SO}_{4}{ }^{2-}$ anions at a concentration higher than $0.1 \mathrm{M}$ can destroy the stability of citrate-capped metal NPs and result in the aggregation of metal NPs ${ }^{24}$ which may be achieved by occupying the surface binding sites of the metal NPs through exchange with the weakly bound citrate ligands. ${ }^{25}$ To further promote the aggregation of the AuNPs, we used $\mathrm{NaHSO}_{4}$ instead of $\mathrm{Na}_{2} \mathrm{SO}_{4}$, because the acidity of the $\mathrm{HSO}_{4}{ }^{-}$salt further promotes the exchange between the $\mathrm{SO}_{4}{ }^{2-}$ anions and citrate ligands due to the weak basicity of citrate. However, the $\mathrm{HSO}_{4}{ }^{-}$anions at a concentration as low as $1.0 \mathrm{mM}$ do not result in the aggregation of the AuNPs at all, but may promote ligand exchange between the strongly-bound berberine molecules and the citrate ligands at the surface of the AuNPs. It is thus expected that the sensitivity of the AuNPs to berberine will be improved by the addition of a suitable amount of $\mathrm{NaHSO}_{4}$ to the AuNP suspension. We studied the effect of different concentrations of $\mathrm{NaHSO}_{4}$ on the AuNPs, and
Fig. 6A shows the absorbance spectra of the AuNP suspension with the addition of different concentrations of $\mathrm{NaHSO}_{4}$. Inset image (a) in Fig. 6A shows the absorbance spectra of the AuNP suspension before and after the addition of $0.1 \mathrm{mM} \mathrm{NaHSO}$. The two absorbance spectra are completely identical, suggesting no aggregation of the AuNPs. Moreover, the $A_{670} / A_{520}$ ratio for the AuNP suspension with $0.1 \mathrm{mM} \mathrm{NaHSO}$ remains at a constant value with time (inset image (b) in Fig. 6A). This clearly indicates that the AuNP suspension is still highly stable after the addition of $0.1 \mathrm{mM} \mathrm{NaHSO}$. We further compared the sensitivities of the AuNPs to berberine before and after the addition of $\mathrm{NaHSO}_{4}$ by measuring the $A_{670} / A_{520}$ at different analyte concentrations. As shown in Fig. $6 \mathrm{~B}$, the $A_{670} / A_{520}$ ratios for the $\mathrm{NaHSO}_{4}$-optimized AuNPs in the presence of 0.5-3.0 $\mu \mathrm{M}$ berberine are obviously larger than the corresponding ratios for the original AuNPs. Moreover, the difference becomes larger with the increase of berberine concentration in the range of 0.5$2.5 \mu \mathrm{M}$, as shown in Fig. 6B. For example, while the $A_{670} / A_{520}$ ratios at 2.0, 2.5 and 3.0 $\mu \mathrm{M}$ berberine for the original AuNPs are only $0.23,0.53$ and 1.09 , respectively, the corresponding ratios for the $\mathrm{NaHSO}_{4}$-optimized AuNPs are 0.99, 1.21 and 1.25, respectively. That is to say, the extinction ratio was enhanced 24 -fold in the low concentration range of the analyte by the addition of $\mathrm{NaHSO}_{4}$. Therefore, the addition of $\mathrm{NaHSO}_{4}$ can significantly improve the aggregation sensitivity of the AuNPs to trace levels of berberine.

\section{Detection of berberine in real samples}

To demonstrate whether the citrate-stabilized AuNPs can be used for the direct detection of berberine in human plasma, we first doped human plasma with $100 \mu \mathrm{L}$ of berberine $(1 \mathrm{mM})$, and then the human plasma was extracted according to the method mentioned in the ESI. $\uparrow$ The extract contains $169.77 \mu \mathrm{g} \mathrm{mL}$ berberine with a recovery rate of $50.47 \%$ from the as-added berberine, and was finally diluted 5 times for the colorimetric
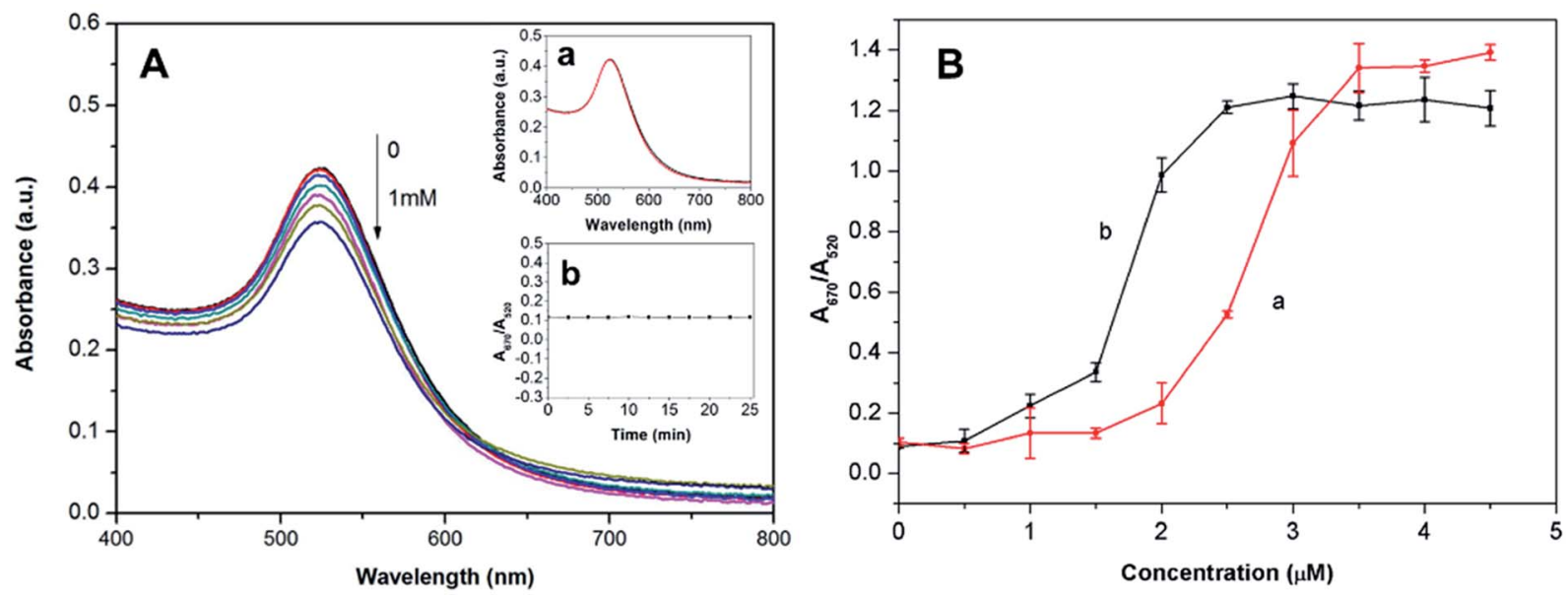

Fig. 6 Optimized sensitivity of colloidal AuNPs with $\mathrm{NaHSO}_{4}$. (A) Absorbance spectra of the colloidal AuNPs after the addition of NaHSO 4 at concentrations of $0,0.1,0.2,0.3,0.5,0.8,1 \mathrm{mM}$. Inset (a) shows the absorbance spectra of the colloidal AuNPs before (black line) and after (red line) the addition of $0.1 \mathrm{mM} \mathrm{NaHSO}_{4}$. Inset (b) is the temporal evolution of the $A_{670} / A_{520}$ values after the addition of $0.1 \mathrm{mM} \mathrm{NaHSO}(0-25 \mathrm{~min}$ ). (B) Evolution of the $A_{670} / A_{520}$ values of the AuNPs with different berberine concentrations before (a) and after (b) the addition of $0.1 \mathrm{mM} \mathrm{NaHSO}_{4}$. 
assay. Meanwhile, a blank extract was also obtained using an identical procedure to evaluate the reliability of the colorimetric assay. The UV-vis spectra also confirm that the $\mathrm{NaHSO}_{4}$-optimized AuNPs are highly sensitive and selective to the extracts of human plasma. With the addition of the blank extract to the AuNP suspension, the absorbance at $520 \mathrm{~nm}$ is slightly reduced, but the absorbance at $670 \mathrm{~nm}$ shows no increase (Fig. 7A), indicating that the aggregation of the AuNPs did not significantly occur and the decrease in absorbance at $520 \mathrm{~nm}$ mainly resulted from the dilution of the AuNPs after the addition of the blank extract. With the addition of the extract from berberinecontaining human plasma, however, the absorbance at $520 \mathrm{~nm}$ obviously reduced and the new absorbance at $670 \mathrm{~nm}$ violently increased with the amount of extract (Fig. 7B). When the final concentration of berberine reached $1.86 \mu \mathrm{g} \mathrm{mL}{ }^{-1}$, the absorbances at $670 \mathrm{~nm}$ are higher than the absorbances at $520 \mathrm{~nm}$. As shown in Fig. 8, the corresponding color of the AuNPs remained constant with the addition of the blank extract (upper image in Fig. 8); however, it can be seen with the naked eye that the color of the AuNPs with the addition of the real sample changed from the original wine-red to blue with different concentrations of berberine (bottom image in Fig. 8). This colorimetric assay allows the detection of concentrations as low as $0.24 \mu \mathrm{g} \mathrm{mL}^{-1}$ with rapid and reliable visual detection of berberine by comparison with a blank sample. Furthermore, there is a linear relationship between the absorbance change of the AuNPs at $520 \mathrm{~nm}$ (Fig. 9) and the concentration of berberine in the real samples in the range of $0.24-0.86 \mu \mathrm{g} \mathrm{mL} L^{-1}\left(R^{2}=\right.$ 0.97). Therefore, it can be concluded that our AuNP-based colorimetric probe can be used for the quantitative analysis of berberine in human plasma and the LOD is $0.06 \mu \mathrm{g} \mathrm{mL}^{-1}$ by UVvis spectroscopy.

As far as we know, there is no defined concentration level of berberine in clinical samples at present. However, several groups have reported the berberine concentration in human and animal blood determined by other methods. Hua et al. ${ }^{26}$

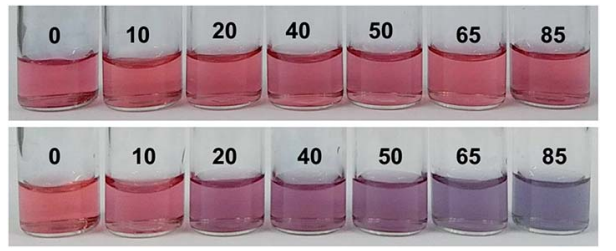

Fig. 8 Visual color changes of the $\mathrm{NaHSO}_{4}$-optimized AuNP sensor after the addition of the extracts from (top) blank human plasma and (bottom) berberine-containing human plasma (the indicated amounts of extract correspond to final berberine concentrations of $0,0.24 \mu \mathrm{g}$ $\mathrm{mL}^{-1}, 0.47 \mu \mathrm{g} \mathrm{mL}^{-1}, 0.91 \mu \mathrm{g} \mathrm{mL}^{-1}, 1.13 \mu \mathrm{g} \mathrm{mL}^{-1}, 1.45 \mu \mathrm{g} \mathrm{mL}^{-1}$, and $1.86 \mu \mathrm{g} \mathrm{mL}^{-1}$, respectively).

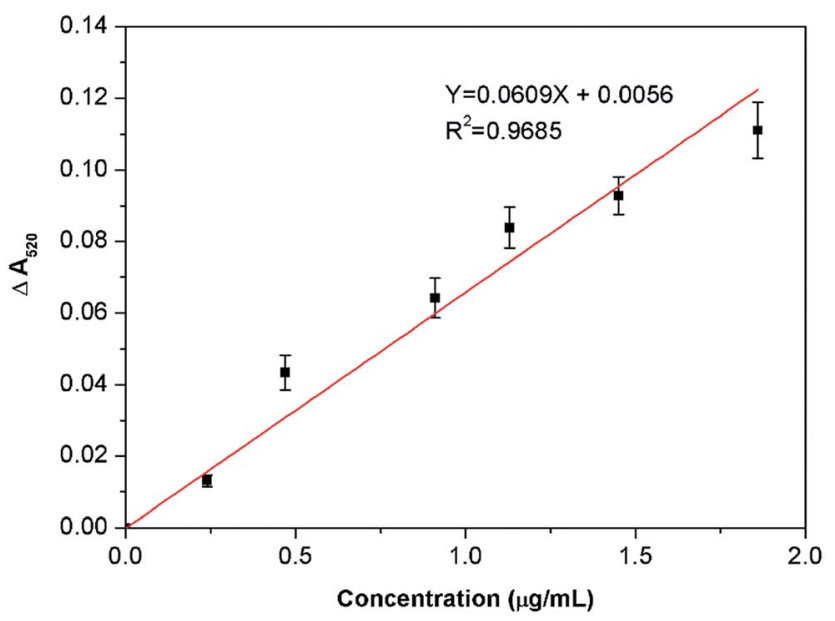

Fig. 9 Linear relationship between the absorbance change of the AuNP suspension at $520 \mathrm{~nm}$ and the concentration of berberine in real samples.

reported that after a $400 \mathrm{mg}$ oral dose of berberine, the mean maximum plasma concentration $\left(C_{\max }\right)$ in twenty volunteers was about $0.4 \mathrm{ng} \mathrm{mL} \mathrm{mL}^{-1}$ determined by a liquid chromatography-
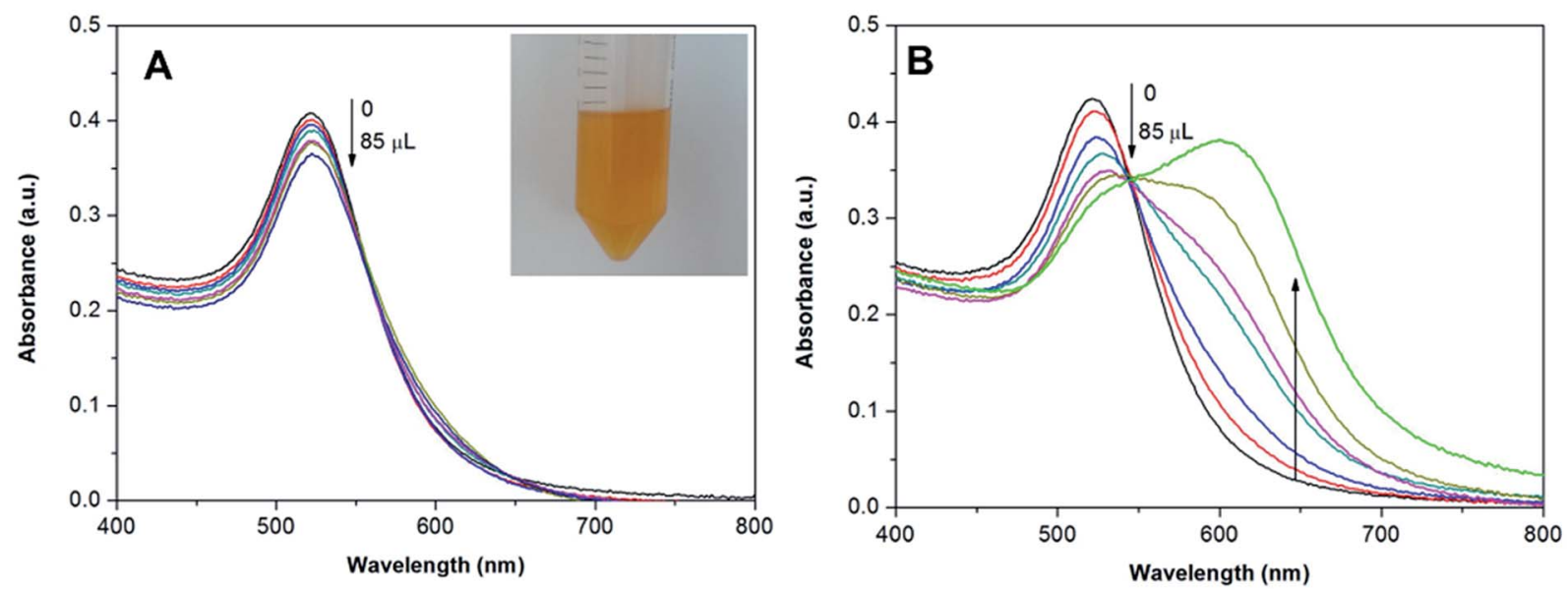

Fig. 7 UV-vis spectra of the $\mathrm{NaHSO}_{4}$-optimized AuNP sensor ( $2 \mathrm{~mL}$ ) after the addition of $0,10,20,40,65$, and $85 \mu \mathrm{L}$ of the extracts from (A) blank human plasma (the inset is a photo of blank human plasma) and (B) berberine-containing human plasma (the extract contains $33.95 \mu \mathrm{g} \mathrm{mL}^{-1}$ berberine; final concentrations are $0,0.24 \mu \mathrm{g} \mathrm{mL}^{-1}, 0.47 \mu \mathrm{g} \mathrm{mL}^{-1}, 0.91 \mu \mathrm{g} \mathrm{mL}^{-1}, 1.13 \mu \mathrm{g} \mathrm{mL}^{-1}, 1.45 \mu \mathrm{g} \mathrm{mL}^{-1}$, and $1.86 \mu \mathrm{g} \mathrm{mL}{ }^{-1}$, respectively). The data were collected after $10 \mathrm{~min}$. 
electrospray ionization-mass spectrometry (LC-ESI-MS) method. Gui et $a .^{27}$ studied the pharmacokinetics of berberine in the blood of rats. The results showed that the $C_{\max }$ of berberine in blood reached $4.169 \mu \mathrm{g} \mathrm{mL} \mathrm{m}^{-1}$ after the oral administration of a microemulsion, and $0.394 \mu \mathrm{g} \mathrm{mL}^{-1}$ after the oral administration of tablets. Such a concentration is apparently higher than the limit of detection that we have achieved in human plasma $\left(0.06 \mu \mathrm{g} \mathrm{mL}^{-1}\right)$.

Although the $C_{\max }$ of berberine in twenty volunteers is much lower than the limit of detection achieved in our method due to the poor absolute bioavailability of orally administered berberine, our colorimetric method is fast, simple and visual compared to the LC-ESI-MS method.

\section{Conclusions}

In summary, we take advantage of the color change of asprepared or optimized citrate-stabilized AuNPs to develop a nanoparticle sensor for simple and reliable visual detection of berberine in human plasma. Due to the negative charge on the surface of the AuNPs, berberine can serve as an inducer to directly induce the aggregation of the unmodified AuNPs without the aid of a salt. This colorimetric method offers several advantages over current berberine detection techniques. First, the method does not require expensive and complicated instruments, which simplifies the operation and reduces the cost. Second, the method allows the detection of concentrations as low as $0.24 \mu \mathrm{g} \mathrm{mL}^{-1}$ to be achieved with the naked eye within $10 \mathrm{~min}$, and therefore it is very suitable for the rapid and ultrasensitive detection of berberine in human plasma. Finally, this sensor exhibits excellent selectivity for berberine over other molecules with positive charges and similar structures and the residual ingredients in the extracts from human plasma. These advantages make this method quite promising for on-site and real-time detection of berberine in human blood for clinical applications.

\section{Conflict of interest}

The authors declare no competing financial interests.

\section{Acknowledgements}

This work was supported by the National Natural Science Foundation of China $(21505053,21405138)$, and the Science and Technology Planning Project of Guangdong Province, China (2015A030401045, 2016A030310089).

\section{References}

1 X. M. Yang, J. S. Wang, J. Luo and L. Y. Kong, One-step largescale preparative isolation of isoquinoline alkaloids from rhizoma coptidis chinensis by polyamide column chromatography and their quantitative structure-retention relationship analysis, J. Liq. Chromatogr. Relat. Technol., 2012, 35, 1842-1852.
$2 \mathrm{X}$. Zeng and X. Zeng, Relationship between the clinical effects of berberine on severe congestive heart failure and its concentration in plasma studied by HPLC, Biomed. Chromatogr., 1999, 13, 442-444.

3 Y. W. Wang, X. Yi, K. Ghanam, S. C. Zhang, T. T. Zhao and X. M. Zhu, Berberine decreases cholesterol levels in rats through multiple mechanisms, including inhibition of cholesterol absorption, Metab., Clin. Exp., 2014, 63, 11671177.

4 H. L. Tan, K. G. Chan, P. Pusparajah, A. Duangjai, S. Saokaew, T. M. Khan, L. H. Lee and B. H. Goh, Rhizoma Coptidis: A potential cardiovascular protective agent, Front. Pharmacol., 2016, 7, 13.

5 S. Habtemariam, Berberine and inflammatory bowel disease: A concise review, Pharmacol. Res., 2016, 113, 592-599.

6 C. Q. Chen, M. L. Lu, Q. H. Pan, J. Fichna, L. J. Zheng, K. S. Wang, Z. Yu, Y. Y. Li, K. Li, A. H. Song, Z. C. Liu, Z. S. Song and M. Kreis, Berberine improves intestinal motility and visceral pain in the mouse models mimicking diarrhea-predominant irritable bowel syndrome (ibs-d) symptoms in an opioid-receptor dependent manner, PLOS One, 2015, 10, 14.

7 W. B. Zha, G. Liang, J. Xiao, E. J. Studer, P. B. Hylemon, W. M. Pandak, G. J. Wang, X. K. Li and H. P. Zhou, Berberine inhibits HIV protease inhibitor-induced inflammatory response by modulating ER stress signaling pathways in murine macrophages, PLoS One, 2010, 5, 8.

8 T. Y. Wu, F. R. Chang, J. R. Liou, I. W. Lo, T. C. Chung and L. Y. Lee, Rapid HPLC quantification approach for detection of active constituents in modern combinatorial formula, San-Huang-Xie-Xin-Tang (SHXXT), Front. Pharmacol., 2016, 7, 1-15.

9 L. Yang, X. Meng, X. Yu and H. Kuang, Simultaneous determination of anemoside $\mathrm{B} 4$, phellodendrine, berberine, palmatine, obakunone, esculin, esculetin in rat plasma by UPLC-ESI-MS/MS and its application to a comparative pharmacokinetic study in normal and ulcerative colitis rats, J. Pharm. Biomed. Anal., 2017, 134, 43-52.

10 Z. W. Yuan, E. L. H. Leung, X. X. Fan, H. Zhou, W. Z. Ma, L. Liu and Y. Xie, Quantitative evaluation of berberine subcellular distribution and cellular accumulation in nonsmall cell lung cancer cells by UPLC-MS/MS, Talanta, 2015, 144, 20-28.

11 S. Liang, Y. F. Kuang, F. F. Ma, S. Chen and Y. F. Long, A sensitive spectrofluorometric method for detection of berberine hydrochloride using $\mathrm{Ag}$ nanoclusters directed by natural fish sperm DNA, Biosens. Bioelectron., 2016, 85, 758-763.

12 Y. Q. Guo, Y. X. Chen, Y. L. Wei, H. H. Li and C. Dong, Labelfree fluorescent aptasensor for potassium ion using structure-switching aptamers and berberine, Spectrochim. Acta, Part A, 2015, 136, 1635-1641.

$13 \mathrm{~J} . \mathrm{Li}, \mathrm{L}$. Zhang and $\mathrm{X}$. Lin, Rapid and nondestructive detection of berberine hydrochloride in tablets using optical fiber sensing with integrating sphere reflection, $J$. Anal. Sci., 2015, 31, 285-287. 
14 K. L. Ai, Y. L. Liu and L. H. Lu, Hydrogen-bonding recognition-induced color change of gold nanoparticles for visual detection of melamine in raw milk and infant formula, J. Am. Chem. Soc., 2009, 131, 9496-9497.

15 H. Chi, B. H. Liu, G. J. Guan, Z. P. Zhang and M. Y. Han, A simple, reliable and sensitive colorimetric visualization of melamine in milk by unmodified gold nanoparticles, Analyst, 2010, 135, 1070-1075.

16 Z. Q. Gao, Z. L. Qiu, M. H. Lu, J. Shu and D. P. Tang, Hybridization chain reaction-based colorimetric aptasensor of adenosine $5^{\prime}$-triphosphate on unmodified gold nanoparticles and two label-free hairpin probes, Biosens. Bioelectron., 2017, 89, 1006-1012.

17 K. Tian, G. Siegel and A. Tiwari, A simple and selective colorimetric mercury(II) sensing system based on chitosan stabilized gold nanoparticles and 2,6-pyridinedicarboxylic acid, Mater. Sci. Eng., C, 2017, 71, 195-199.

18 J. Ling, Y. Sang and C. Z. Huang, Visual colorimetric detection of berberine hydrochloride with silver nanoparticles, J. Pharm. Biomed. Anal., 2008, 47, 860-864.

19 M. C. Daniel and D. Astruc, Gold nanoparticles: Assembly, supramolecular chemistry, quantum-size-related properties, and applications toward biology, catalysis, and nanotechnology, Chem. Rev., 2004, 104, 293-346.

20 D. D. Evanoff and G. Chumanov, Size-controlled synthesis of nanoparticles. 2. Measurement of extinction, scattering, and absorption cross sections, J. Phys. Chem. B, 2004, 108, 1395713962.
21 K. C. Grabar, R. G. Freeman, M. B. Hommer and M. J. Natan, Preparation and characterization of Au colloid monolayers, Anal. Chem., 1995, 67, 735-743.

22 J. B. Liu, S. H. Fu, B. Yuan, Y. L. Li and Z. X. Deng, Toward a universal "adhesive nanosheet" for the assembly of multiple nanoparticles based on a protein-induced reduction/decoration of graphene oxide, J. Am. Chem. Soc., 2010, 132, 7279-7280.

23 Z. Zhang, H. Sun, X. Shao, D. Li, H. Yu and M. Han, Threedimensionally oriented aggregation of a few hundred nanoparticles into monocrystalline architectures, $A d v$. Mater., 2005, 17, 42-47.

24 X. X. Han, G. G. Huang, B. Zhao and Y. Ozaki, Label-free highly sensitive detection of proteins in aqueous solutions using surface-enhanced Raman scattering, Anal. Chem., 2009, 81, 3329-3333.

25 S. E. J. Bell, N. M. S. Sirimuthu, S. E. J. Bell and N. M. S. Sirimuthu, Surface-enhanced Raman spectroscopy (SERS) for sub-micromolar detection of DNA/RNA mononucleotides, J. Am. Chem. Soc., 2007, 128, 15580-15581.

26 W. Y. Hua, L. Ding, Y. Chen, B. Gong, J. C. He and G. L. Xu, Determination of berberine in human plasma by liquid chromatography-electrospray ionization-mass spectrometry, J. Pharm. Biomed. Anal., 2007, 44, 931-937.

27 S. Y. Gui, L. Wu, D. Y. Peng, Q. Y. Liu, B. P. Yin and J. Z. Shen, Preparation and evaluation of a microemulsion for oral delivery of berberine, Pharmazie, 2008, 63, 516-519. 